to mention our allegiance to psychoeducation as researchers and therapists. We tried to minimise its influence by masking both the doctors in charge of TAU and the outcome assessors.

Fifth, Biswas et al advise that we examine effect modifiers and moderators. In our paper we explain that we did examine one strong empirically supported candidate variable in this regard, namely expressed emotion. ${ }^{1}$ And we failed to confirm its role as effect modifier or moderator.

Last but not least, unfortunately we must confess that we do not fully understand how the authors' proposed 'two-level randomisation' or psychoeducation to 'enhance the maintenance of treatment effects following drug withdrawal' might work. We are more than willing to continue this discussion in order to 'creatively tackle design issues when conducting drug-behaviour trials'.

1 Mino $Y$, Shimodera S, Inoue S, Fujita $H$, Tanaka S, Kanazawa S. Expressed emotion of families and the course of mood disorders: a cohort study in Japan. J Affect Disord 2001; 63: 43-9.

Shinji Shimodera, Department of Neuropsychiatry, Kochi Medical Schoo Kochi, Japan. Email: shimodes@kochi-u.ac.jpa; Kae Shimazu, Department of Neuropsychiatry, Kochi Medical School, Kochi; Atsushi Nishida, Department of Schizophrenia Research, Tokyo Institute of Psychiatry, Tokyo, and Department of Psychiatry, Mie University Graduate School of Medicine, TSu, Mie. Naoto Kamimura, Department of Neuropsychiatry, Kochi Medical School; Hirokazu Fujita, Department of Neuropsychiatry, Kochi Medical School; Shimpei Inoue, Department Department of Neuropsychiatry, Kochi Medical School; Shimpei Inoue, Department
of Neuropsychiatry, Kochi Medical School, Kochi; Toshi A. Furukawa, Department of Health Promotion and Human Behaviour, Kyoto University Graduate School of Medicine/School of Public Health, Kyoto, Japan

doi: 10.1192/bjp.199.2.165a

\section{Understanding the neuroprotective mechanisms of lithium may have clinical significance}

The article by Forlenza et $\mathrm{al}^{1}$ is a useful addition to the literature. Disease-modifying drugs for dementia, and in particular Alzheimer's disease, are sorely needed. Despite very strong preclinical science, translational studies have been relatively limited, so this sort of interventional trial is welcome.

The authors highlight the inhibition of glycogen synthase kinase-3 beta (GSK-3B), a serine/threonine kinase involved in the regulation of numerous intracellular signalling pathways, as the likely mechanism for any neuroprotective effects. Although it is true that there is a literature supporting this pathway, other potential disease-modifying pathways are influenced by lithium. For example, up-regulation of autophagy, an intracellular protein degradation pathway which is able to degrade mutant proteins associated with neurodegeneration, can rescue a variety of animal models of neurodegenerative disease. ${ }^{2}$ In fact, GSK-3B inhibition inhibits autophagy via its effect on the mTOR (mammalian target of rapamycin) pathway. Despite this, lithium ultimately induces autophagy via a dominant mechanism involving inositol monophsphatase inhibition. ${ }^{3}$ These distinctions are not trivial, as understanding the interactions of these pathways allows for more rational treatment design. For example, lithium and rapamycin (a drug which inhibits $\mathrm{mTOR}$ ) provides greater neuroprotection in fly models of Huntington's disease than either drug alone. ${ }^{4}$ Furthermore, numerous US Food and Drug Administration-approved drugs which are autophagy up-regulators have been identified. Many of these may have a more favourable side-effect profile than lithium, and preclinical work suggests their efficacy in animal models of neurodegenerative disease. ${ }^{5}$

The potential mechanisms for neuroprotection by lithium extend well beyond inhibition of GSK-3B. Working out which are most important is of more than scientific interest as it is likely to allow rational drug design and better selection of currently available drugs with neuroprotective potential.

1 Forlenza OV, Diniz BS, Radanovic M, Santos FS, Talib LL, Gattaz WF. Disease-modifying properties of long-term lithium treatment for amnestic mild cognitive impairment: randomised controlled trial. Br J Psychiatry 2011; 198: $351-6$.

2 Garcia-Arencibia M, Hochfeld W, Toh P, Rubinsztein DC. Autophagy, a guardian against neurodegeneration. Semin Cell Dev Biol 2010; 7: 691-8.

3 Sarkar S, Floto RA, Berger Z, Imarisio S, Cordenier A, Pasco M, et al. Lithium induces autophagy by inhibiting inositol monophosphatase. J Cell Biol 2005; 7: $1101-11$

4 Sarkar S, Krishna G, Imarisio S, Saiki S, O'Kane CJ, Rubinsztein DC. A rational mechanism for combination treatment of Huntington's disease using lithium and rapamycin. Hum Mol Genet 2008; 2: 170-8.

5 Williams A, Sarkar S, Cuddon P, Ttofi EK, Saiki S, Siddiqi FH, et al. Novel targets for Huntington's disease in an mTOR-independent autophagy pathway. Nat Chem Biol 2008; 5: 295-305.

Benjamin R. Underwood, Consultant Psychiatrist, Suffolk Mental Health Partnership NHS Trust. Email: ben.underwood@smhp.nhs.uk

doi: 10.1192/bjp.199.2.166

Authors' reply: We agree with the comments by Dr Underwood reinforcing that the mechanisms by which lithium may exert a neuroprotective effect in patients with amnestic mild cognitive impairment ${ }^{1}$ still must be clarified. The inhibition of glycogen synthase kinase-3 (GSK-3B) by lithium is a plausible effect, given its pivotal role in the pathogenesis of Alzheimer's disease, but most likely not the only one. In addition to the prevailing mechanism of action involving the inhibition of inositol monophosphatase and downstream effects towards the up-regulation of autophagy, many other neurobiological effects have been attributed to lithium. These include the inhibition of apoptosis and the up-regulation of neurotrophic cascades. ${ }^{2}$ The modification of these intracellular signalling systems by lithium has been shown to yield neurotrophic and/or neuroprotective effects, which have been consistently demonstrated in cell culture and animal models. These effects are probably unspecific and may be beneficial to patients with distinct psychiatric and neurodegenerative diseases, including bipolar disorder, ${ }^{3}$ amyotrophic lateral sclerosis ${ }^{4}$ and Alzheimer's disease. ${ }^{1}$

We hypothesise that the inhibition of GSK-3B by lithium is more specific to processes that ultimately lead to the formation of neuritic plaques and neurofibrillary tangles. According to the 'GSK3 hypothesis of Alzheimer's disease', overactive GSK-3B accounts for memory impairment, Tau hyperphosphorylation, increased beta-amyloid production and local plaque-associated inflammatory responses mediated by the microglia. ${ }^{5}$ The inhibition of GSK-3B is currently regarded as a candidate disease-modifying approach for the treatment and prevention of Alzheimer's disease, and specific inhibitors such as NP-031112 are being tested in phase II clinical trials (www.clinicaltrials.gov). Therefore, lithium may contribute to the attenuation of the pathological process in Alzheimer's disease through inhibition of GSK-3B, and deliver additional, unspecific benefits via modification of other signalling pathways that favour autophagy, preclude apoptosis and up-regulate the secretion of neurotrophic factors in the brain. Presumably, the interplay of complementary mechanisms is necessary to warrant clinically relevant benefits, which we were able to show in our study. ${ }^{1}$ We thus speculate that the effects of lithium on multiple homeostatic systems downstream from membrane receptor-based neurotransmission may in fact represent an advantage as a candidate drug for the treatment of complex neurobiological diseases. In our study, the doses of lithium used were very well tolerated. This, 
together with its wide availability and low cost, warrant the further investigation of the potential protective effect of lithium in Alzheimer's disease.

Declaration of interest

Funding for the present work provided by Conselho Nacional de Pesquisa Científica (CNPq, Project 554535/2005-0), Alzheimer's Association (NIRG-08-90688), Fundação de Amparo à Pesquisa do Estado de São Paulo (FAPESP, Project 02/13633-7) and Associação Beneficente Alzira Denise Hertzog da Silva (ABADHS).

1 Forlenza OV, Diniz BS, Radanovic M, Santos FS, Talib LL, Gattaz WF. Disease-modifying properties of long-term lithium treatment for amnestic mild cognitive impairment: randomised controlled trial. Br J Psychiatry 2011; 198: $351-6$

2 de Sousa RT, van de Bilt MT, Diniz BS, Ladeira RB, Portela LV, Souza DO, et al. Lithium increases plasma brain-derived neurotrophic factor in acute bipolar mania: a preliminary 4-week study. Neurosci Lett 2011; 494 $54-6$.

3 Nunes PV, Forlenza OV, Gattaz WF. Lithium and risk for Alzheimer's disease in elderly patients with bipolar disorder. Br J Psychiatry 2007; 190: 359-60.

4 Aggarwal SP, Zinman L, Simpson E, McKinley J, Jackson KE, Pinto H, et al. Safety and efficacy of lithium in combination with riluzole for treatment of amyotrophic lateral sclerosis: a randomised, double-blind, placebo-controlled trial. Lancet Neurol 2010; 9: 481-8.

5 Hooper C, Killick R, Lovestone S. The GSK3 hypothesis of Alzheimer's disease. J Neurochem 2008; 104: 1433-9.

Orestes V. Forlenza, Laboratory of Neuroscience (LIM-27), Department and Institute of Psychiatry, Faculty of Medicine, University of São Paulo, Rua Dr. Ovídio Pires de Campos 785, 05403-010, São Paulo, SP, Brazil. Email: forlenza@usp.br; Breno S. Diniz, Wagner F. Gattaz, Laboratory of Neuroscience (LIM-27), Department and Institute of Psychiatry, Faculty of Medicine, University of São Paulo, Brazil

doi: 10.1192/bjp.199.2.166a 